\title{
Pulsed electric field technology in food preservation:
} a review

\begin{abstract}
Thermal inputs for food preservation generally dominate the food processing industry. However such treatments may lead to several undesirable changes in quality attributes and nutritional value of food. On the contrary, non-thermal preservation methods have a minimal impact on the sensory quality and nutritional status of food. Several non-thermal techniques have been involved in food preservation with an objective to maintain the nutritional properties and physic-chemical characteristics of food products. In this regard, pulsed electric field provides an alternative choice for various food products, particularly for liquid foods, for better preservation and maintenance of fresh-like quality aspects of food. Correspondingly, this memoir highlights the potential of pulsed electric field technology as an effective tool to preserve various foods with special reference to microbial inactivation. Additionally, principles, associated mechanistic approaches, effect of this treatment on food quality and problems linked with the consumer acceptance are also discussed in this script.
\end{abstract}

Volume 6 Issue 6 - 2017

\author{
Qamar Abbas Syed, Anum Ishaq, Ubaid Ur \\ Rahman, Sadia Aslam, Rizwan Shukat \\ National Institute of Food Science and Technology, University of \\ Agriculture, Pakistan
}

Correspondence: Qamar Abbas Syed, National Institute of Food Science and Technology, Faculty of Food, Nutrition and Home Sciences, University of Agriculture, Faisalabad, Pakistan, Email Qamar.Abbas@uaf.edu.pk

Received: September 26, 2016 | Published: June 19, 2017

Keywords: pulsed electric field, non-thermal preservation, food quality, microbial inactivation

\section{Introduction}

Traditional food processing approaches like cooking, freezing, refrigeration and blanching are commonly known methods and are generally used by the people in their homes. However, in the food processing industries these techniques are combined with other processing operations with the help of several contemporary processtechnology plants by using different technical prospects that are not commonly practiced by consumers during household processing. Modern food technology deals with the development of conventional processing methods such as high-temperature short time heating or vacuum cooking, as well as several other approaches like high pressure treatment, extrusion, pulsed electric field technology and microwave processing. These modern technologies usually emphasize on food preservation with maximum retention of food quality attributes and nutrients. Therefore the frequently used concept of "minimal processing" is not absolutely apt, since actually the principle "as little as possible, but as much as necessary" is meant. ${ }^{1}$

A wide range of non-thermal processing techniques have gained popularity in the recent times as a potential tool for the substitution or replacement of traditional thermal processing methods of foods. Additionally, non-thermal processes offer several advantages over thermal processes such as low processing temperatures, efficient energy utilization, keeping the quality of food like color, flavor, taste $\&$ nutrient retention and inactivation of quality deteriorative enzymes $\&$ spoilage causing microorganisms. ${ }^{2}$

Pulsed electric field (PEF) technology is a non-thermal food preservation method that involves the use of short electricity pulses for microbial inactivation while imposing minimal detrimental influence on food quality. This technology has the major advantage to provide high-quality foods to the consumers. PEF is claimed as superior to thermal processing and preservation methods because it reduces detrimental changes in food quality and nutrition and keeps physical and sensorial attributes of food. ${ }^{3}$
PEF technology has wide range of applications ranging from liquid or semi-solid foods to solid foods. Most PEF studies have captivated on application of high voltage pulses for microbial inactivation milk and dairy products, egg products, juices and other liquid foods. ${ }^{4}$ However, most researchers have mainly focused on the aspect of food preservation with special reference to the microbial control and a lesser information is presented about the effect of PEF on food composition, quality and acceptability. Recently, some investigations have been conducted to evaluate the potential of PEF for improving food processing efficiency like enhancement of juice extraction and escalation of the food dehydration or drying. ${ }^{5}$

Wouters et al., ${ }^{6}$ reported that PEF is known as one of the most auspicious non-thermal tools for microbial decontamination of foods. It involves the generation of electric fields $(5-50 \mathrm{kV} / \mathrm{cm})$ with the help of short high voltage pulses ( $\mu \mathrm{s})$ between two electrodes that leads to microbial inactivation at temperatures lower than thermal methods. They also proposed that exact mechanistic approach of microbial inactivation is not known however, it is normally hypothesized that PEF causes penneabilization or depolarization of microbial membranes.

Studies revealed that PEF technology enables inactivation of bacterial and yeast vegetative cells in various foods. On the contrary, bacterial spores cannot be killed by employing pulsed electric fields because spores are resistant to PEF. Thus, applications of PEF are primarily focused on pathogenic and spoilage causing microorganisms in food. In addition to the pronounced effect of this technology in controlling microbiological spoilage of foods in a rapid and uniform manner, PEF also delivers shelf life extension without using heat treatment and preservation of sensorial and nutritional quality of foods. Likewise, PEF is also capable to improve the energy usage in an efficient and economical way. Hence, successful applications of PEF technology propose an alternative to conventional thermal processing techniques for food preservation and processing. ${ }^{7}$ In this article, principles, mechanisms and recent applications of PEF technology 
are reviewed and compared with thermal processing technologies. Furthermore, examples are coded to illustrate the potential of PEF technology for aiming at preserving the quality features of various foods.

\section{Principle of PEF}

PEF technology involves the use of pulses having higher electric fields for only a few micro to milliseconds with intensity in the range of $10-80 \mathrm{kV} / \mathrm{cm}$. The process depends on the number of pulses delivered to the product which is held between two electrodes. These electrodes have a specific gap between them which is known as treatment gap of the chamber. During PEF processing, high voltage is applied that results in the inactivation of microorganisms present in the food sample. The electric field is applied in different forms like as exponentially decaying waves, bipolar waves or oscillatory pulses. The process can also be carried at various temperature ranges such as ambient, sub-ambient and above-ambient temperatures. Food is packed after treatment with PEF and then stored under refrigerated conditions.

The science involved behind the transfer of electric pulses from food is that food contains several ions that provide a definite level of electrical conductivity to the product. This technique is usually preferred for liquid foods because electrical current flows into the liquid food more efficiently and the transfer of pulses from one point to other in liquids is quite easy due to the presence of charged molecules present. ${ }^{8}$

A group of researchers, Zimmermann et al., ${ }^{9}$ stated that mechanism of the functioning of PEF technology is the delivery of pulsing power to the product that is placed between a set of electrodes confining the treatment gap of the PEF chamber. The typical system has a pulse generator that produces high voltage pulses, treatment chamber that handles the product to be treated and associated with controlling and monitoring devices. Food product is placed in the treatment chamber equipped with electrodes connected with each other by a nonconductive material which prevents the flow of electricity from one to the other High voltage electrical pulses are generated that are transferred to the product. The product placed between the electrodes experiences a force per unit charge which ruptures the bacterial cell membranes. ${ }^{10}$ In general, PEF technology is suggested for pasteurization of various food products including milk, juices, yogurt, liquid eggs and soups. ${ }^{11}$ Furthermore, combination of PEF with ultrasound, high pressure and ultraviolet light treatments may enhance the process output.

\section{Microbial inactivation by PEF}

Several studies have investigated the mode of action of PEF to reduce the microbial load in various food products. Nonetheless, the exact mechanistic approach underlying the microbial inactivation by PEF has not been fully expounded as yet. However, a general mechanism of PEF action involves instability of microbial membranes by the induction of electrical field and electromechanical compression that leads to the pore formation in membrane. ${ }^{12}$ Mechanical fickleness of membranes is caused due to critical membrane potential which is formed by electrical field. Electroporation results in a significant increase in the membrane rupture and permeability which is termed as electro permeabilization. Electropermeabilization can be reversible or irreversible, depending on the degree of membrane organizational changes that results in cell death. ${ }^{13}$ Literature explains that membrane permeability is increased in a considerable manner by increasing the strength of electric field. This elevated membrane instability harmonizes with inactivation of microbial cells. In general, spores are claimed as more obstinate to PEF treatment as compared to vegetative cells. $^{14}$

\section{Factors influencing PEF performance to inactivate microorganisms}

The capability of PEF to inactivate microbes depends on several factors that can be categorized as process parameters, nature of product and properties of microbial cells. ${ }^{6}$ These factors play a dynamic role to attain the optimum performance of PEF treatment. An overview of these parameters is discussed herein.

\section{Process parameters}

Several process parameters affect the ability of PEF to reduce microbial population in food such as strength of electric field, pulse length and shape, number of pulses and temperature. ${ }^{15}$ On a general node, increased intensity of these factors improves microbial inactivation but their exact relationship with the survival rate of microorganisms is not clear. Therefore, exact measurement of all these parameters is required to acquire reliable results.

\section{Product nature}

The administration of PEF is also influenced by nature of product as a vast range of products are being treated by PEF which include fruit juices, liquid eggs, milk and dry herbs. It is investigated through experimental trials that PEF treatment is not much effective for products having particles or special structures, i.e. emulsions. ${ }^{6}$ Additionally, physical and chemical properties of the food also affect the rate of microbial decontamination. Various studies have revealed the influence of $\mathrm{pH}$, water activity and electrical conductivity on the efficiency of PEF to inactivate microorganisms. ${ }^{16}$

$\mathrm{pH}$ has a significant influence on the inactivation kinetics of microbes. Jeantet et al., ${ }^{17}$ reported that higher inactivation of Salmonella was observed in foods having neutral or above neutral $\mathrm{pH}$ values. Similarly, Alvarez et al., ${ }^{16}$ testified substantial reduction in L. monocytogenes number in high acid foods such as citrus juices. Likewise, conductivity of the treatment medium has inverse relation with microbial inactivation. It is observed that foods having high electrical conductivity show less inactivation of microorganisms after PEF treatment..$^{18}$ On the contrary, water activity has a direct relation with microbial reduction by PEF treatment as confirmed by Min \& Zhang. ${ }^{19}$

\section{Characteristics of microbes}

Inactivation of microorganism by employing PEF technology also depends on microbial characteristics including type of microorganisms, species and strains. ${ }^{20}$ Generally, Gram-positive and Gram-negative bacteria are thought to be more resistant in comparison with yeast cells. Likewise, bacterial and mold spores are also claimed as recalcitrant to PEF treatment. ${ }^{14}$ Additionally, cell size and shape also affect the inactivation kinetics due to the difference in the development of critical membrane potential. ${ }^{21}$ PEF treatment affects different bacterial species at altered rate. It is usually proposed that Salmonella and E. coli are more susceptible to PEF as compared to Listeria and Bacillus species. Growth conditions like temperature, growth medium, concentration of nutrients and $\mathrm{pH}$ of the treated medium also influence PEF efficiency as well. ${ }^{6}$ 


\section{Applications in food industry}

Application of PEF technology has been extensively demonstrated for the pasteurization of various food products like juices, milk \& dairy products, soup and liquid eggs. However, it has several limitations such as product must be free from air bubbles and must have lower electrical conductivity. Additionally, particle size should be less than the gap of the treatment region to ensure appropriate treatment. PEF is generally not suitable for solid foods however several solid products have also been investigated to be efficiently treated by deploying PEF treatment. PEF technology can also be used to enhance the extraction of several bioactive components and sugars from plant cells. ${ }^{2}$

PEF processing has shown its potential to treat less viscous fruit juices having less electrical conductivity such as apple, citrus and cranberry juices. PEF technology also executes beneficial aspects on the quality parameters of fruit juice. Correspondingly, Yeom et al., ${ }^{22}$ compared pasteurized and PEF-treated citrus juice during refrigerated storage $\left(4^{\circ} \mathrm{C}\right)$ for a period of 112 days and observed less browning in PEF-processed juice comparatively to traditionally pasteurized juice due to conversion of ascorbic acid to furfural. Besides, PEFtreated foods also retained their fresh flavor, textural \& functional attributes and extended shelf life in addition with microbiological safety. ${ }^{23}$ In recent years, PEF technology has been utilized for various purposes like enhancement of drying efficiency, modification of enzymatic activity, solid food preservation, waste water treatment and extraction..$^{24}$

\section{PEF in fruit processing}

PEF processing has promising applications in citrus industry with special reference to the inactivation of microorganisms and prevention of developing off-flavors during the storage. ${ }^{25}$ Jemai et al., ${ }^{21}$ noticed that treatment of apple juice with PEF resulted in enhancement of diffusion coefficients of soluble substances. This technology can also be fruitfully applied for the disintegration of biological tissues that enhances the extraction of intracellular compounds from different fruits. For instance, pectin is a very useful component found in many fruits that is traditionally being extracted through enzymatic reaction but this reaction has less yield of pectin due to poor efficiency. Alternatively, PEF treatment is employed as short pulses to avoid excessive heat and undesirable electrolytic reactions that can enhance the extraction rate of pectin from fruit pomace. ${ }^{26}$

\section{Bacterial inactivation in milk by PEF}

Inadequately pasteurized milk may cause several health hazards due to the presence of several spoilage causing and pathogenic bacteria particularly Escherichia coli and Listeria and Pseudomonas spp. ${ }^{27}$ Elevated concerns regarding the impact of heat treatment on the quality of milk and consumer demand for fresh-like quality attribute products have encouraged the induction of PEF for milk pasteurization. PEF-induced microbial inactivation is believed to be an effective way of milk preservation without adversely affecting the milk quality.

Studies have demonstrated the effectiveness of PEF processing for microbial reduction in simulated milk ultra-filtrate and skim milk. ${ }^{28}$ However, presence of fat and protein moieties limits the adeptness of PEF in whole milk because these molecules protect bacterial cells during treatment. ${ }^{29}$ Therefore, it is imperative to validate the worth of PEF treatment to inactivate bacteria in a complex whole milk matrix for a genuine comparison with thermal pasteurization.
Garcia et al., ${ }^{30}$ observed sub-lethal injury in bacterial cells and concluded that PEF treatment can be successfully used in synergy with other hurdles to get more benefits. In another trial, PEF processing of milk was combined with heat treatment up to $55-60^{\circ} \mathrm{C}$ and a significant reduction was observed in the microbial load. ${ }^{31}$ More recently, Sharma et al. ${ }^{29}$ employed PEF treatment at different dose, time and temperature ranges to inactivate Gram-positive and Gramnegative bacteria in whole milk and reported 5-6 log reduction in bacterial number at $22-28 \mathrm{kV} \mathrm{cm}^{-1}$ for $17-101 \mu \mathrm{s}$ at $50^{\circ} \mathrm{C}$. On the other hand, some studies did not clearly demonstrate the effects pre-heating, temperature, time \& dose variation on PEF efficiency and effects of PEF treatment on milk quality and functional properties. Therefore, it is questionable whether PEF can maintain the integrity of heatsensitive milk components while inactivating spoilage and pathogenic microorganisms in whole milk and need further exploration.

\section{PEF and meat quality}

Meat products have been widely consumed around the globe due to the presence of valuable micronutrients high quality protein. ${ }^{32}$ Quality of meat is at prime importance because meat quality is considered as the most vital factor for purchasing decisions of consumers. ${ }^{33}$ Pulsed electric field technology has shown its potential for various applications in solid foods with different aims including structural modifications, changing physical quality parameters, extraction of bioactive compounds and preservation. Nevertheless, this technology has limited applications in muscle foods. ${ }^{34} \mathrm{PEF}$ technology can be employed in meat processing for various purposes including enhancement of cell permeation to increase tenderness, attenuation of microbial load to improve the shelf life and maintenance of volatile profile of meat during storage. ${ }^{35}$

PEF technology can considerably improve enzyme release and glycolysis that are essential for proteolysis for meat tenderization. O'Dowd et al., ${ }^{34}$ studied the effect of PEF on meat quality @ $1.1-2.8 \mathrm{kV}$ $\mathrm{cm}^{-1}$ and reported no significant improvement in meat tenderness during storage. Later on, Bekhit et al., ${ }^{36}$ found a weighty improvement in beef tenderness by PEF treatment $(5-10 \mathrm{kV})$. Likewise, ${ }^{37}$ applied PEF $(5-10 \mathrm{kV})$ at different frequencies $(20,50$ and $90 \mathrm{~Hz})$ on beef muscles and revealed that PEF treatment reduced shear force up to $19 \%$ \& improved tenderness by augmenting the degradation of desmin and troponin-T during a refrigeration storage of 21days.

\section{Consumer acceptance of PEF treated products}

Pulsed electric field technology is gaining importance as a nonthermal way to process or preserve foods. Additionally, industrial implementation of this technique and commercialization of PEFtreated food products are also in progress. Conversely, there is a lack of steadiness in terms of terminology, product \& process description, product communication with consumer and marketability of the PEFprocessed foods. Efficient marketability of an emerging technology needs unambiguous benefits for consumers. Consumer attitude determines the level of acceptance of a novel food product and this attitude depends on the method of introduction of a new product and technology. ${ }^{38}$

PEF-processed foods have limited acceptance by the consumers because of several reasons. The major hitch is that the nature of this technology has not yet fully expressed to consumers as consumers require information about the products they consume. Moreover, PEFtreated products are generally labeled as "minimally processed foods" that induces negative image in the mind of consumer that the food is 
not well-processed and may cause serious health implications after consumption. ${ }^{39}$ Since clear communication is of prime importance for consumers, the description about the technology and terminologies used on the labeling should be explained to them in order to enhance the marketability of PEF-processed foods.

\section{Conclusion}

Pulsed electric field technology is a quite effective approach for the preservation and processing of a variety of food products without much affecting their quality attributes. Though, research on PEF as a non-thermal technique should not be limited only towards microbial inactivation but also towards the retardation of several spoilage inducing chemical and enzymatic reactions, retention of functional components in food during and after treatment. Although data is available in literature regarding the efficiency of PEF processing on enzyme inactivation, enhancement of proteolysis in protein enriched foods and its effects on some quality characteristics of food, however, detailed research work is needed to explore the mechanisms involved behind these functionalities. In addition, proper planning is needed to boost up the marketability of PEF-processed foods by providing unambiguous information to consumers through proper product labeling and conducting commercialization campaigns.

\section{Acknowledgements}

None.

\section{Conflict of interest}

The author declares no conflict of interest.

\section{References}

1. Reineke K, Schottroff F, Meneses N, et al. Sterilization of liquid foods by pulsed electric fields-an innovative ultra-high temperature process. Front Microbiol. 2015;6:400.

2. Siemer C, Aganovic K, Toepfl S, et al. Application of Pulsed Electric Fields in Food. Conventional and Advanced Food Processing Technologies. 2015. p. 645-672.

3. Quass DW. Pulsed electric field processing in the food industry. A status report on pulsed electric field. Electric Power Research Institute, California, USA; 1997. p. 23-35.

4. Qin BL, Zhang Q, Barbosa-Cánovas G, et al. Pulsed electric field treatment chamber design for liquid food pasteurization using finite element method. Trans ASAE. 1995;38:557.

5. Vorobiev E, Jemai AB, Bouzrara $\mathrm{H}$, et al. Pulsed electric field assisted extraction of juice from food plants. Novel Food Processing Technologies, New York, USA; 2004. p. 105-130.

6. Wouters P, Alvarez I, Raso I. Critical factors determining inactivation kinetics by pulsed electric field food processing. Trends in Food Science \& Technology. 2001;12(3-4):112-121.

7. Hodgins A, Mittal G, Griffiths M. Pasteurization of fresh orange juice using low-energy pulsed electrical field. Journal of Food Science. 2002;67(6):2294-2299.

8. Zhang QH, Barbosa-Cánovas GV, Swanson BG. Engineering aspects of pulsed electric field pasteurization. Journal of Food Engineering. 1995;25(2):261-281.

9. Zimmermann U, Benz R. Dependence of the electrical breakdown voltage on the charging time in Valonia utricularis. The Journal of Membrane Biology. 1980;53(1):33-43.
10. Fernandez-Diaz MD, Barsotti L, Dumacy E, et al. Effects of pulsed electric fields on ovalbumin solutions and dialyzed egg white. J Agric Food Chem. 2000;48(6):2332-2339.

11. Bendicho S, Barbosa-Canovas GV, Martin O. Reduction of protease activity in simulated milk ultrafiltrate by continuous flow high intensity pulsed electric field treatments. Journal of Food Science. 2003;68(3):952-957.

12. Barbosa-Canovas GV, Gongora MM, Pothakamury UR, et al. Preservation of foods with pulsed electric fields. Academic Press, San Diego, USA; 1999

13. Rowan NJ, Mac Gregor SJ, Anderson JG, et al. Pulsed electric inactivation of diarrhoeagenic Bacillus cereus through irreversible electroporation. Letters in Applied Microbiology. 2000;31(2):110-114.

14. Katsuki S, Majima T, Nagata $\mathrm{K}$, et al. Inactivation of Bacillus strearothermophilus by pulsed electric field. IEEE Transactions on Plasma Science. 2000;28(1):155-160.

15. Raso J, Alvarez I, Condon S, et al. Predicting inactivation of Salmonella senftenberg by pulsed electric fields. Innovative Food Science \& Emerging Technologies. 2000;1(1):21-29.

16. Alvarez I, Raso J, Palop A, et al. Influence of different factors on the inactivation of Salmonella senftenberg by pulsed electric fields. International Journal of Food Microbiology. 2000;55(1-3):143-146.

17. Jeantet R, Baron F, Nau F, et al. High intensity pulsed electric fields applied to egg white: effect on Salmonella enteritidis inactivation and protein denaturation. J Food Prot. 1999;62(12):1381-1386.

18. Wouters PC, Dutreux N, Smelt JPPM, et al. Effects of pulsed electric fields on inactivation kinetics of Listeria innocua. Appl Environ Microbiol. 1999;65(12):5364-5371.

19. Min S, Zhang H. Effect of water activity on the inactivation of Enterobacter cloacae by pulsed electric field treatment. IFT Annual Meeting, Dallas, USA; 2000

20. MacGregor SJ, Farish O, Fouracre R, et al. Inactivation of pathogenic and spoilage microorganisms in a test liquid using pulsed electric fields. IEEE Transactions on Plasma Science. 2000;28(1):144-149.

21. Qiu X, Sharma S, Tuhela L, et al. An integrated PEF pilot plant for continuous nonthermal pasteurization of fresh orange juice. Trans ASAE. 1998;41(4):1069-1074.

22. Yeorn H, Streaker C, Zhang Q, et al. Effects of pulsed electric fields on the quality of orange juice and comparison with heat pasteurization. $J$ Agric Food Chem. 2000;48(10):4597-4605.

23. Dunn J. Pulsed electric field processing:An overview, In: BarbosaCanovas G, et al. editors. Pulsed Electric Fields in Food Processing, Fundamental Aspects and Applications Technomic Press, Lancaster, Pennsylvania, USA; 2001. p. 1-30.

24. Ade-Omowaye BIO, Taiwo K, Knorr D. Use of pulsed electric field pretreatment to improve dehydration characteristics of plant based foods. Trends Food Sci Technol. 2002;12:285-295.

25. Hendrix CM, Red JB. Chemistry and technology of citrus juices and byproducts. Production and Packaging of Non-Carbonated Fruit Juices and Fruit Beverages. 1995. p. 53-87.

26. Min S, Evrendilek GA, Zhang QH. Pulsed electric fields:processing system, microbial and enzyme inhibition, and shelf-life extension of foods. IEEE Transactions on Plasma Science. 2007;35(1):59-73

27. Ercolini D, Russo F, Ferrocino I, et al. Molecular identification of mesophilic and psychrotrophic bacteria from raw cow's milk. Food Microbiol. 2009;26(2):228-231. 
28. Sobrino-López A, Martín-Belloso O. Review: potential of highintensity pulsed electric field technology for milk processing. Food Engineering Reviews. 2010;2(1):17-27.

29. Sharma P, Bremer P, Oeya I, et al. Bacterial inactivation in whole milk using pulsed electric field processing. International Dairy Journal. 2014;35(1):49-56

30. Garcia D, Gomez N, Manas P, et al. Occurrence of sublethal injury after pulsed electric fields depending on the microorganism, the treatment medium $\mathrm{pH}$ and the intensity of the treatment investigated. Journal of Applied Microbiology. 2005;99:94-104.

31. Guerrero-Beltran JA, Sepulveda DR, Gongora-Nieto MM, et al. Milk thermization by pulsed electric fields and electrically induced heat. Journal of Food Engineering. 2010;100(1):56-60.

32. Pereira PM, Vicente AF. Meat nutritional composition and nutritive role in the human diet. Meat Sci. 2013;93(3):586-592.

33. Bolumar T, Enneking M, Toepfl S, et al. New developments in shockwave technology intended for meat tenderization:Opportunities and challenges. A review. Meat Sci. 2013;95(4):931-939.

34. O'Dowd LP, Arimi JM, Noci F, et al. An assessment of the effect of pulsed electrical fields on tenderness and selected quality attributes of post rigor beef muscle. Meat Science. 2013;93(2):303-309.
35. Faridnia F, Ma QL, Bremer PJ, et al. Effect of freezing as pre-treatment prior to pulsed electric field processing on quality traits of beef muscles. Innovative Food Science \& Emerging Technologies. 2015;29:31-40.

36. Bekhit AED, Van de Ven R, Suwandy V, et al. Effect of pulsed electric field treatment on cold-boned muscles on different potential tenderness. Food Bioprocess Technol; 2014.

37. Suwandy V, Carne A, Van de Ven R, et al. Effect of pulsed electric field on the proteolysis of cold boned beef M. Longissimus lumborum and M. Semimembranosus. Meat Sci. 2015;100:222-226.

38. Jaeger H, Knorr D, Szao E, et al. Impact of terminology on consumer acceptance of emerging technologies through the example of PEF technology. Innovative Food Science and Emerging Technologies. 2015;29:87-93.

39. Cardello AV, Schutz HG, Lesher LL. Consumer perceptions of foods processed by innovative and emerging technologies: A conjoint analytic study. Innovative Food Science \& Emerging Technologies. 2007;8(1):73-83. 